\title{
Gemella asaccharolytica sp. nov., isolated from human clinical specimens
}

\author{
Nurver Ulger-Toprak, ${ }^{1,2}$ Paula H. Summanen, ${ }^{1}$ Chengxu Liu, ${ }^{1}$ \\ Marie-Claire Rowlinson ${ }^{1}$ and Sydney M. Finegold ${ }^{3,4}$ \\ ${ }^{1}$ Research Service, VA Medical Center West Los Angeles, CA, USA \\ ${ }^{2}$ Marmara University, Faculty of Medicine, Department of Microbiology, Istanbul, Turkey \\ ${ }^{3}$ Infectious Diseases Section, VA Medical Center West Los Angeles, CA, USA \\ ${ }^{4}$ Departments of Medicine and of Microbiology, Immunology, and Molecular Genetics, UCLA School \\ of Medicine, Los Angeles, CA, USA
}

Correspondence

Sydney M. Finegold

sidfinegol@aol.com
Gemella species are catalase-negative, facultatively anaerobic, Gram-variable cocci, arranged in pairs, tetrads, clusters and sometimes short chains. Some strains decolorize quite readily in the Gram stain, and may appear Gram-negative (Berger, 1992). In addition, some strains may require strictly anaerobic conditions for primary isolation and become aerotolerant after transfer to laboratory media (Facklam \& Elliott, 1995). Four species isolated from human sources are recognized, Gemella haemolysans, the type species of the genus, Gemella morbillorum, Gemella bergeri and Gemella sanguinis (Collins et al., 1998a). Both G. haemolysans and G. morbillorum are residents of the mucous membranes of humans and of certain warm-blooded animals and are recognized opportunistic pathogens. Although the habitats of G. bergeri and G. sanguinis are unknown, it seems probable that these species also form part of the normal human microbiota (Collins et al., 1998b). Two additional Gemella species isolated from animal sources have been described: Gemella palaticanis was isolated from the oral cavity of a dog and Gemella cuniculi from the submandibular abscess of a rabbit (Collins et al., 1999; Hoyles et al., 2000). We have isolated three Gemella-like isolates from three different human wound specimens. Comparative $16 \mathrm{~S}$ rRNA gene sequencing studies demonstrated that the

The GenBank/EMBL/DDBJ accession number for the 16S rRNA gene sequence of strain WAL $1945 J^{\top}$ is EU427463. unknown strains were genealogically homogeneous but did not correspond to any of the recognized species of the genus Gemella. In this article we report the phenotypic characteristics of the organism and the results of a phylogenetic analysis.

Three novel bacterial strains, WAL 1822, from an arm wound in an intravenous drug abuser patient, WAL $1945 \mathrm{~J}^{\mathrm{T}}$, from an infection following a cut finger in an immunocompetent patient, and WAL 1990F, from a labial abscess in a cancer patient, and reference strains G. haemolysans CCUG $37985^{\mathrm{T}}$, G. morbillorum CCUG $15561^{\mathrm{T}}$, G. bergeri CCUG $37817^{\mathrm{T}}$ and G. sanguinis CCUG $37820^{\mathrm{T}}$ were included in the present study. The novel strains were isolated from mixed infections at the VA Wadsworth Anaerobe Laboratory from clinical specimens obtained at Olive View-UCLA Hospital. The specimens were collected, transported and processed as outlined by Jousimies-Somer et al. (2002). Anaerobic conditions consisted of a gas mixture of $5 \% \mathrm{CO}_{2}, 5 \% \mathrm{H}_{2}$ and $90 \%$ $\mathrm{N}_{2}$. Microaerophilic conditions were obtained using an automated evacuation-replacement system (Anoxomat; microaerophilic cycle). All testing was done on $24-72 \mathrm{~h}$ pure cultures on plates of Brucella blood agar (Anaerobe Systems), CDC blood agar (Becton Dickinson Microbiology) or trypticase soy blood agar (TSBA; Becton Dickinson Microbiology). Single colonies were selected, described and subcultured to assure purity and to determine whether the organism was aerobic, anaerobic or 
microaerophilic. $\beta$-Lactamase testing was done using nitrocefin disks (Cefinase; Becton Dickinson).

The strains were characterized biochemically by using a combination of conventional tests as described by Jousimies-Somer et al. (2002) and the commercially available biochemical Rapid ID 32A, Rapid ID 32 Strep, API 20 Strep and API ZYM kits (bioMérieux). The commercial biochemical kits were used according to the manufacturer's instructions and the results were graded using the colour chart supplied by the manufacturer. All biochemical tests were performed in duplicate. Carbohydrate fermentation tests were performed using prereduced, anaerobically sterilized (PRAS) biochemical broth tubes (Anaerobe Systems). The strains were grown in peptone-yeast-glucose (PYG) broth (Anaerobe Systems) for metabolic end-product analysis (short-chain volatile and non-volatile fatty acids) by GLC.

The 16S rRNA genes were amplified by PCR using universal primers 8UA (positions 8-28, Escherichia coli numbering) and 1485B (positions 1485-1507) as described previously (Song et al., 2003). The amplified product was purified by using a QIAamp PCR purification kit (Qiagen, Inc.) and directly sequenced with an ABI 3100 Avant Genetic System (Applied Biosystems). The closest known relatives of the new isolates were determined by performing database searches using the BLAST software (Benson et al., 1997). Almost the full length of the $16 \mathrm{~S}$ rRNA gene sequences $(>1400 \mathrm{nt})$ of the unidentified bacteria and of closely related bacteria were aligned using CLUSTAL W (Thompson et al., 1994). The phylogenetic tree was constructed by the CLUSTAL $\mathrm{w}$ method using Lasergene 7 SeqBuilder and MEGALIGN programs (DNASTAR, Inc.). Bootstrap analysis was performed using the Lasergene 7 MEGALIGN program.

The novel strains recovered from clinical infections were isolated together with other anaerobes or aerobes. WAL $1822 \mathrm{M}$ was isolated together with strains of Staphylococcus aureus, Streptococcus anginosus, Escherichia coli, Klebsiella pneumoniae, Parvimonas micra, Peptoniphilus asaccharolyticus, Peptoniphilus harei and Bacteroides ureolyticus; WAL $1945 \mathrm{~J}^{\mathrm{T}}$ with strains of Kluyvera ascorbata, Enterobacter cloacae, Finegoldia magna, Peptostreptococcus anaerobius, Dialister microaerophilus, Slackia exigua, Veillonella species and Prevotella species; and WAL 1990F with strains of Gardnerella vaginalis, Micrococcus luteus and Peptoniphilus asaccharolyticus. Moderate to heavy growth was obtained for most of the isolates on primary isolation blood agar plates. The novel isolates were present at $\geqslant 10^{5}$ c.f.u. $\mathrm{ml}^{-1}$ (WAL $1822 \mathrm{M}, 1 \times 10^{8}$ c.f.u. $\mathrm{ml}^{-1}$; WAL $1945 \mathrm{~J}^{\mathrm{T}}, 2 \times 10^{5}$ c.f.u. $\mathrm{ml}^{-1}$; WAL $1990 \mathrm{~F}, 5 \times 10^{8}$ c.f.u. $\mathrm{ml}^{-1}$ ). Typical cells of all the novel strains were found to be Gram-variable cocci that occurred singly, in pairs or in short chains. Colonies on Brucella blood agar plates at 5 days were lowconvex, circular, entire, non-pigmented and semi-translucent with a diameter of $0.4-0.5 \mathrm{~mm}$ and surrounded by a zone of greening ( $\alpha$-haemolysis). They all grew well anaerobically, and ultimately in atmospheres of 2 and $6 \% \mathrm{O}_{2}$ and $5 \% \mathrm{CO}_{2}$. All strains were sensitive to special-potency identification discs containing kanamycin $(1000 \mu \mathrm{g})$, sodium polyanethol sulfonate (SPS; $1000 \mu \mathrm{g})$ and vancomycin $(5 \mu \mathrm{g})$ and resistant to colistin sulfate $(10 \mu \mathrm{g})$ discs. $\beta$-Lactamase production was negative.

The novel strains were negative for catalase, urease and nitrate reduction, failed to grow in broth containing $6.5 \%$ $\mathrm{NaCl}$ and were non-motile. The unknown strains were all asaccharolytic; they did not produce acid from glucose or other carbohydrates. The four reference strains produced acid from a few sugars; all fermented glucose and mannose. G. morbillorum CCUG $15561^{\mathrm{T}}$ gave positive reactions for acid production from fructose, maltose and sucrose. G. bergeri CCUG $37817^{\mathrm{T}}$ also fermented fructose, as well as mannitol. G. haemolysans CCUG $37985^{\mathrm{T}}$ and G. sanguinis CCUG $37820^{\mathrm{T}}$ produced acid from maltose and sucrose, respectively. In PYG broth, moderate amounts of acetic acid and small amounts of lactic acid were produced by all three novel isolates and the four reference strains. Using Rapid ID 32A, all three novel isolates produced the same profile. Positive reactions were obtained for arginine arylamidase, proline arylamidase, leucyl glycine arylamidase, phenylalanine arylamidase, leucine arylamidase, tyrosine arylamidase, glycine arylamidase, histidine arylamidase and serine arylamidase. All the other tests were negative. The novel strains and G. bergeri CCUG $37817^{\mathrm{T}}$ were pyrrolidonyl arylamidase-negative, but the other known strains were positive. According to the API ZYM system, all unknown isolates had esterase and esterase lipase activity and they also produced leucine arylamidase and naphthol-AS-BI-phosphohydrolase. Production of acid phosphatase and alkaline phosphatase was negative for the novel isolates and for $G$. bergeri CCUG $37817^{\mathrm{T}}$ and G. morbillorum CCUG $15561^{\mathrm{T}}$, but was positive for G. haemolysans CCUG $37985^{\mathrm{T}}$ and G. sanguinis CCUG $37820^{\mathrm{T}}$. In the Rapid ID 32 Strep system, the novel strains produced positive reactions for pyroglutamic acid arylamidase and hippurate; alanyl-phenylalanyl-proline arylamidase and glycyl-tryptophan arylamidase and all other reactions were negative. Using API 20 Strep, all strains produced leucine aminopeptidase and hydrolysed hippurate. The Voges-Proskauer reaction was negative for all strains except for G. sanguinis CCUG $37820^{\mathrm{T}}$. None hydrolysed aesculin or gelatin. Tests that serve to distinguish the novel bacterium from other Gemella species are given in Table 1.

To assess the genealogical affinity between the unknown bacteria and their relationship with other Gram-positive, catalase-negative taxa, 16S rRNA gene sequences were determined. Pairwise analysis showed that the three novel isolates were phylogenetically closely related to each other ( $>99 \%$ sequence similarity). Sequence searches of the GenBank and Ribosomal Database Project libraries for species with validly published names revealed that the unknown organisms were phylogenetically most closely associated with species of the genus Gemella (approx. 92- 
Table 1. Characteristics useful in differentiating Gemella asaccharolytica sp. nov. from other Gemella species

Species: 1, G. asaccharolytica sp. nov.; 2, G. bergeri; 3, G. cuniculi; 4, G. haemolysans; 5, G. morbillorum; 6, G. palaticanis; 7, G. sanguinis. +, Positive; - , negative; $\mathrm{v}$, variable; $+^{-}$, most strains positive; ${ }^{-}$, some strains may display weak activity. Based on the current study and on data from Collins et al. (1998a, b, 1999) and Hoyles et al. (2000). ND, No data available.

\begin{tabular}{|c|c|c|c|c|c|c|c|}
\hline Characteristic & 1 & 2 & 3 & 4 & 5 & 6 & 7 \\
\hline \multicolumn{8}{|l|}{ Acid production from: } \\
\hline Glucose & - & + & + & + & + & + & + \\
\hline Lactose & - & - & - & - & - & + & - \\
\hline Mannitol & - & $\mathrm{V}$ & + & - & $\mathrm{V}$ & - & + \\
\hline Maltose & - & - & - & + & + & + & $+^{-}$ \\
\hline Sucrose & - & - & - & $\mathrm{V}$ & + & + & + \\
\hline Sorbitol & - & - & + & - & $\mathrm{V}$ & - & + \\
\hline Trehalose & - & - & - & - & - & + & - \\
\hline \multicolumn{8}{|l|}{ Production of: } \\
\hline Alkaline phosphatase & - & - & + & + & - & - & + \\
\hline Acid phosphatase & - & $-\mathrm{w}$ & + & + & $-\mathrm{w}$ & $\mathrm{ND}$ & + \\
\hline $\begin{array}{l}\text { Alanyl-phenylalanyl-proline } \\
\text { arylamidase }\end{array}$ & - & - & - & - & $\mathrm{V}$ & + & $\mathrm{V}$ \\
\hline Glycyl-tryptophan arylamidase & - & - & - & $\mathrm{V}$ & $\mathrm{V}$ & + & - \\
\hline Hippurate hydrolysis & + & - & - & - & $-\mathrm{w}$ & - & - \\
\hline
\end{tabular}

$94 \%$ sequence similarity). A UPGMA tree depicting the phylogenetic affinity of the unknown coccus with closely related taxa is shown in Fig. 1. The unknown coccus clustered within the Gemella clade, forming a distinct subline.

The polyphasic taxonomic analysis shows clearly that the novel coccus-shaped bacterium isolated from human wound infections represents an as-yet unrecognized member of the genus Gemella. 16SrRNA gene sequence divergence values of more than $6 \%$ from other members of this genus demonstrate unequivocally that the unknown coccus is worthy of separate species status. In addition, the unidentified coccus can be readily distinguished phenotypically from all currently described Gemella species by biochemical tests (Table 1). Therefore, based on both phylogenetic and phenotypic evidence, we consider that the bacterium merits classification as a novel species of the genus Gemella, for which the name Gemella asaccharolytica sp. nov. is proposed.

\section{Description of Gemella asaccharolytica sp. nov.}

Gemella asaccharolytica (a.sac'cha.ro.ly'ti.ca. Gr. pref. a- not; Gr. n. saccharon sugar; N.L. fem. adj. lytica from Gr. adj. lutikos able to lyse, able to loose; N.L. fem. adj. asaccharolytica not digesting sugar).

Cells stain Gram-variable and are non-motile cocci, approximately $0.5 \mu \mathrm{m}$ in diameter. Colonies on Brucella blood agar plates at 5 days are small, smooth, convex, circular, entire, semi-translucent, $0.4-0.5 \mathrm{~mm}$ in diameter and surrounded by a zone of greening ( $\alpha$-haemolysis). Non-pigmented. Grows under strictly anaerobic conditions and after a number of subcultures in the presence of 2 and $6 \% \mathrm{O}_{2}$ and $5 \% \mathrm{CO}_{2}$. Negative for catalase and oxidase activity, indole production and nitrate reduction. Asaccharolytic; acid is not produced from glucose or the following other carbohydrates: adonitol, amygdalin, arabinose, cellobiose, erythritol, fructose, galactose, glycogen, inositol, inulin, lactose, maltose, mannitol, mannose, melezitose, melibiose, raffinose, rhamnose, ribose, salicin, sorbitol, sucrose, trehalose, xylose and xylan. In PYG broth, moderate amounts of acetic acid and small amounts of lactic acid are produced. Esterase lipase, esterase (weak), naphthol-AS-BI-phosphohydrolase, arginine arylamidase, proline arylamidase, leucyl glycine arylamidase, phenylalanine arylamidase, leucine arylamidase, tyrosine arylamidase, glycine arylamidase, histidine arylamidase, serine arylamidase and pyroglutamic acid arylamidase are produced. Hippuric acid is hydrolysed. Acid phosphatase, alkaline phosphatase, alanyl-phenylalanyl-proline arylamidase, glycyl-tryptophan arylamidase, pyrrolidonyl arylamidase, urease and trypsin are not produced. Aesculin, starch and gelatin are not hydrolysed. Voges-Proskauer test is negative. Sensitive to vancomycin $(5 \mu \mathrm{g})$, kanamycin $(1000 \mu \mathrm{g})$ and SPS $(1000 \mu \mathrm{g})$ and resistant to colistin sulfate $(10 \mu \mathrm{g})$ special-potency identification discs. $\beta$-Lactamase-negative.

The type strain, WAL $1945 \mathrm{~J}^{\mathrm{T}} \quad\left(=\mathrm{ATCC}\right.$ BAA- $1630^{\mathrm{T}}$ $=$ CCUG $57045^{\mathrm{T}}$ ), was isolated from a human wound

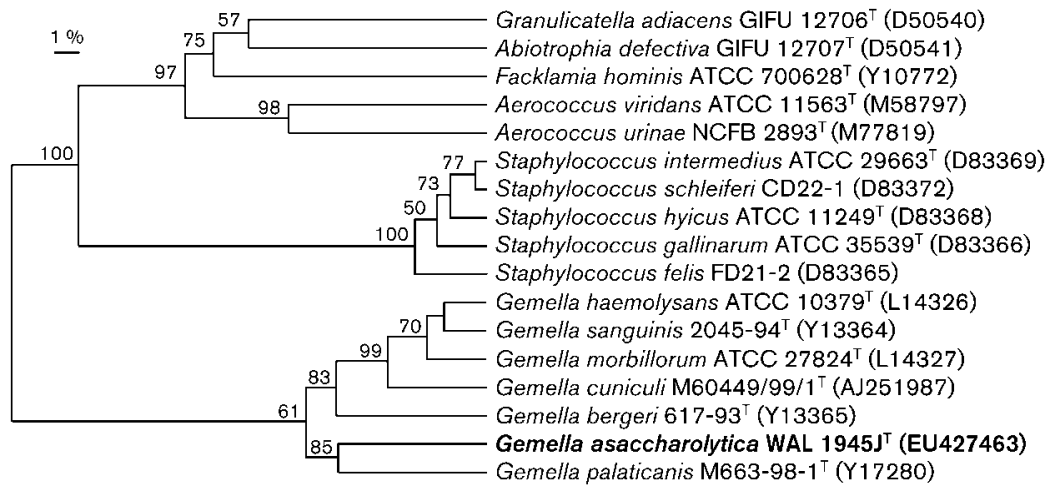

Fig. 1. Unrooted tree showing the phylogenetic relationships of strain WAL $1945 \mathrm{~J}^{\top}$ (Gemella asaccharolytica sp. nov.) with the other related taxa. The UPGMA tree, constructed using CLUSTAL W, was based on a comparison of $16 \mathrm{~S}$ rRNA gene sequences of approximately $1400 \mathrm{nt}$. Bootstrap values, expressed as percentages of 1000 replications, are given at branching points. Bar, $1 \%$ sequence divergence. 
infection. Habitat is unknown. Strains WAL 1822M and WAL 1990F are additional strains of the species.

\section{Acknowledgements}

This work has been supported by DOD grant no. W81XWH-0510134 and by VA Merit Review funds.

\section{References}

Benson, D. A., Boguski, M. S., Lipman, D. J. \& Ostell, J. (1997). GenBank. Nucleic Acids Res 25, 1-6.

Berger, U. (1992) The genus Gemella. In The Prokaryotes, 2nd edn, pp. 1643-1653. Edited by A. Balows, H. G. Trüper, M. Dworkin, W. Harder \& K. H. Schleifer. New York: Springer.

Collins, M. D., Hutson, R. A., Falsen, E., Sjöden, B. \& Facklam, R. R. (1998a). Gemella bergeriae sp. nov., isolated from human clinical specimens. J Clin Microbiol 36, 1290-1293.

Collins, M. D., Hutson, R. A., Falsen, E., Sjöden, B. \& Facklam, R. R. (1998b). Description of Gemella sanguinis sp. nov., isolated from human clinical specimens. J Clin Microbiol 36, 3090-3093.
Collins, M. D., Rodriguez Jovita, M., Foster, G., Sjödén, B. \& Falsen, E. (1999). Characterization of a Gemella-like organism from the oral cavity of a dog: description of Gemella palaticanis sp. nov. Int J Syst Bacteriol 49, 1523-1526.

Facklam, R. \& Elliott, J. A. (1995). Identification, classification, and clinical relevance of catalase-negative, gram-positive cocci, excluding the streptococci and enterococci. Clin Microbiol Rev 8, 479495.

Hoyles, L., Foster, G., Falsen, E. \& Collins, M. D. (2000). Characterization of a Gemella-like organism isolated from an abscess of a rabbit: description of Gemella cuniculi sp. nov. Int J Syst Evol Microbiol 50, 2037-2041.

Jousimies-Somer, H., Summanen, P., Citron, D. M., Baron, E. J., Wexler, H. M. \& Finegold, S. M. (2002). Wadsworth-KTL Anaerobic Bacteriology Manual, 6th edn. Belmont, CA: Star Publishing.

Song, Y., Liu, C., McTeague, M. \& Finegold, S. M. (2003). 16 S ribosomal DNA sequence-based analysis of clinically significant grampositive anaerobic cocci. J Clin Microbiol 41, 1363-1369.

Thompson, J. D., Higgins, D. G. \& Gibson, T. J. (1994). CLUSTAL W: improving the sensitivity for progressive multiple sequence alignment through sequence weighting, position-specific gap penalties and weight matrix choice. Nucleic Acids Res 22, 4673-4680. 